\title{
Estudio descriptivo de algunas variables emocionales en las personas mayores
}

\author{
Igone Etxeberria \\ Matia Instituto Gerontológico (España)
}

\begin{abstract}
Se analizó el funcionamiento emocional (afecto positivo y negativo, satisfacción con la vida, soledad y estrategias de regulación) de las personas centenarias en comparación con el de personas de menor edad (65-74, 75-84 y 85-94 años).

Participaron en el estudio 257 personas mayores sin deterioro cognitivo y sin dependencia para las Actividades de la Vida Diaria (AVD).

Los resultados revelaron que, en las personas centenarias, se producía una disminución de la afectividad positiva en comparación con los grupos de 65 a 74 años y de 75 a 84 años, pero no así con el de 85 a 94 años. Además, presentaban menor afecto negativo y más satisfacción con la vida (en comparación con el grupo de 65 a 74 años), al tiempo que manifestaban sentirse más solas (en comparación con los grupos de 65 a 74 años y de 75 a 84 años). Con respecto a las estrategias de regulación, en general, las personas centenarias utilizan en menor medida las estrategias proactivas y la solución de problemas y en mayor medida las pasivas, al menos en la regulación de la tristeza.

En conclusión, a pesar de experimentar menos afecto positivo y más soledad se produce cierta disminución en el afecto negativo, e incluso cierto aumento de la satisfacción con la vida. En lo que respecta a la regulación emocional, las personas centenarias utilizan menos las estrategias proactivas y más las pasivas (tristeza).
\end{abstract}

Palabras clave: Diferencias de edad, funcionamiento emocional, personas centenarias.

Descriptive study of emotional variables elderly people. Emotional functioning (positive and negative affect, life satisfaction, loneliness and regulation strategies) of centenarians compared to younger adults was analyzed (65-74, 75-84, and 85-94 years old).

This study was conducted with 257 older adults cognitively healthy and independent for the performance of the Activities of Daily Living (ADL).

Results showed a decrease in positive affect in comparison with 65 to 74 and 75 to 84 age groups, but not with 85 to 94 . Centenarians also showed less negative affect and increased life satisfaction (in comparison with the youngest age group). In comparison with 65 to 74 and 75 to 84 age groups centenarians experimented higher loneliness. In regard to regulation strategies, in general, centenarians use less proactive strategies and problem solving while more passive strategies at least when regulating sadness.

In conclusion, despite experiencing less positive affect and a higher loneliness, less negative affect and a higher life satisfaction was observed among centenarians. In addition, centenarians use proactive strategies less and passive strategies more.

Keywords: Age differences, emotional functioning, centenarians.

Correspondencia: Igone Etxeberria. Sorgintxulo, n² 2 izq. C.P.: 20120 Hernani. Guipúzcoa. E mail: ietxeberria1@gmail.com 
Son escasos los trabajos nacionales e internacionales que han centrado su atención en el estudio del funcionamiento emocional en las personas centenarias. Sin embargo, estudiar el funcionamiento emocional en las personas mayores de 85 años tiene gran interés, ya que las enfermedades y limitaciones funcionales, muy prevalentes en estas edades, pueden conllevar que la persona se vuelva más vulnerable en términos emocionales

La revisión bibliográfica se ha focalizado en las variables estudiadas en esta investigación. A pesar de que se ha centrado en encontrar referencias relativas a las personas centenarias, en algunas variables y debido a la escasez de estudios en el tema, se ha recogido la información con respecto a los mayores de 85 años.

\section{Afecto positivo}

La mayoría de estudios apuntan a un descenso de la afectividad positiva con la edad, especialmente, a partir de los 85 años (Baltes y Mayer, 1999). En el caso particular de los centenarios, los escasísimos estudios que han estudiado su funcionamiento emocional indican que se produce un declinar de la afectividad positiva en estas personas (Martin, Da Rosa, Margrett, Garasky, y Franke, 2012). En definitiva, según los datos con los que se cuenta en la actualidad, parece que en las personas centenarias el afecto positivo disminuye. Sin embargo, la escasez de estudios en esta área pone de relieve la necesidad de investigaciones que arrojen luz a los resultados existentes.

\section{Afecto negativo}

Teniendo en cuenta las innumerables pérdidas que sufren las personas muy mayores, a nivel cognitivo, funcional, de salud, a nivel social etc., lo esperable sería que el afecto negativo aumente. Sin embargo, mientras que algunos estudios encontraron niveles más altos de afecto negativo, la mayoría de autores observaron que se produce una estabilidad (Staudinger, Freund, Linden, y Maas 1999), e incluso un decremento de la afectividad negativa en los muy mayores (Stacey y Gatz, 1991).

Ante este estado de la investigación es difícil concretar una hipótesis. No obstante, a partir de nuestra experiencia con personas muy mayores, en este estudio nos inclinamos por una estabilidad o una cierta disminución de la afectividad negativa en dichas personas.

\section{Satisfacción con la vida}

Los resultados de los diversos estudios analizados sobre la satisfacción con la vida en los muy mayores muestra también resultados inconsistentes. Mientras que algunas investigaciones abogan por una estabilidad (Yi y Vaupel, 2002), otras muestran una mayor satisfacción con la vida (Yanguas et al., 2008; Galiana, Gutiérrez, Sancho, Oliver, y Tomás, 2015; Montero, López-Giménez, Acevedo, y Mora, 2015) y otros 
estudios, incluso un decremento (Mroczek y Spiro, 2005). Sin embargo, se ha hallado que controlando ciertas variables (el estado civil, la comorbilidad, las limitaciones funcionales y la soledad), la satisfacción con la vida de los muy mayores es superior a la de mayores más jóvenes (Ailshire y Crimmins, 2011). En este sentido, Aberg, Sidenvall, Hepworth, O'Reilly, y Lithell (2005), han puesto de manifiesto que las personas muy mayores se adaptan a las circunstancias, muchas veces negativas (pérdida de capacidad funcional, cognitiva, y problemas de salud), manteniendo el optimismo y otorgando significado a las experiencias vitales significativas, lo cual redundaría en un mayor bienestar. Atendiendo a este estudio, sería esperable que la satisfacción con la vida en los muy mayores incremente.

\section{Soledad}

Se ha afirmado que en edades avanzadas la pérdida de la red y el apoyo social puede tener especial relevancia en la experimentación de la soledad (Long y Martín, 2000). Por su parte, Pinquart y Sörensen (2001) señalan que los niveles altos de soledad en los muy mayores podrían estar relacionados con la disminución de actividad como consecuencia del deterioro funcional y sensorial que es habitual en edades avanzadas, y no tanto por la falta de contacto social. Tener una movilidad reducida, así como discapacidad sensorial, dificulta el mantenimiento de contactos sociales, lo cual repercute indirectamente en la soledad (Holzschuher, 1984).

En resumen, en la vejez parece existir una relación positiva entre la edad y la soledad; en otras palabras, los muy mayores presentan, en comparación con los mayores más jóvenes, más sentimientos de soledad.

\section{Estrategias de regulación}

Las investigaciones que han estudiado la regulación de las emociones en las personas centenarias son aún más escasas. Una de las pocas referencias indica que, en comparación con sexagenarios y octogenarios, los centenarios utilizaban más estrategias de tipo cognitivo, mientras que los sexagenarios mostraban estrategias de tipo conductual (Martin, Kliegel, Rott, Poon, y Johnson, 2008). En concreto, las personas centenarias tienden a utilizar más las estrategias de tipo pasivo ("no preocuparse", "a rezar", "aceptar los problemas de salud", etc.,) (Poon, Martin, y Margrett, 2010).

En conclusión, teniendo en cuenta el estado de la cuestión, se constata la necesidad de investigar más en conocer el funcionamiento emocional de los muy mayores, y concretamente de las personas centenarias, sobre todo para el tratamiento de las posibles situaciones de vulnerabilidad emocional. Es por ello, que esta investigación tuvo por objetivo conocer cómo es el funcionamiento emocional (afecto positivo y negativo, satisfacción con la vida, soledad y estrategias de regulación) de las personas 
centenarias en comparación con el de personas de menor edad (de 65 a 74 años, de 75 a 84 años, y de 85 a 94 años).

\section{MÉTODO}

\section{Participantes}

En esta investigación participaron 257 personas mayores de 65 años residentes en la provincia de Guipúzcoa. El rango de edad estaba comprendido entre los 65 y los 104 años. Todos ellos eran independientes en las actividades de la vida diaria (AVD) y no presentaban trastornos del estado de ánimo ni deterioro cognitivo asociado a enfermedad neurodegenerativa. La distribución por edades y género se encuentra en la tabla 1 .

\begin{tabular}{lccccc}
\multicolumn{6}{l}{ Tabla 1. Distribución de la muestra por edad y género } \\
\hline $\begin{array}{l}\text { Rango } \\
\text { de edad }\end{array}$ & \multirow{2}{*}{$N$} & $\begin{array}{c}\text { Hombres } \\
N\end{array}$ & $\begin{array}{c}\text { Mujeres } \\
N\end{array}$ & $M$ & $D T$ \\
\hline $65-74$ & 75 & 33 & 42 & 68,77 & 2,66 \\
\hline $75-84$ & 80 & 32 & 48 & 79,20 & 2,93 \\
\hline $85-94$ & 69 & 30 & 39 & 88,86 & 2,72 \\
\hline $95-104$ & 33 & 10 & 23 & 100,21 & 2,16 \\
\hline Total & 257 & 105 & 152 & & \\
\hline
\end{tabular}

\section{Instrumentos}

Escala PANAS de afecto positivo y negativo (Sandín, Chorot, Lostao, Joiner, Santed, y Valiente, 1999): esta escala mide la afectividad positiva y negativa rasgo y estado. Está compuesta por 20 ítems, con un formato de respuesta de 1 a 5.

Escala de satisfacción con la vida (Pons, Atienza, Balaguer, y García-Merita, 2002): este instrumento proporciona una medida de bienestar subjetivo, poniendo énfasis en los aspectos cognitivos y evaluativos del bienestar. Consta de 5 ítems que se puntúan en una escala tipo Likert con recorrido de 1 (totalmente en desacuerdo) hasta 7 (totalmente de acuerdo).

Escala de Soledad UCLA (Vázquez y Jiménez, 1994): se trata de una escala de 20 ítems; la persona ha de señalar la frecuencia con la que experimenta las vivencias descritas en cada uno de los ítems en una escala de 1 a 4.

Cuestionario de estrategias de regulación (Blanchard-Fields y Coats, 2007): se aplicó la versión adaptada (Etxeberria, Etxebarria, Urdaneta, y Yanguas, 2015), por lo que se aplicaron dos situaciones una para la generación de cada una de las emociones (tristeza e ira). El cuestionario está compuesto por 22 ítems que reflejan distintas estrategias de regulación. Abarca 4 factores: expresión emocional y búsqueda de consejo (ambas estrategias de regulación proactivas), estrategias pasivas (evitación, aceptación y supresión) y la solución de problemas. Las respuestas se recogen en una escala tipo Likert, en la que las opciones de respuesta varían de 1=En absoluto a 4=Definitivamente haría eso. 


\section{Procedimiento}

Para efectuar la captación de los participantes más jóvenes (menores de 85 años) se contactó con las juntas directivas de los centros sociales de los pueblos adyacentes a San Sebastián. Sin embargo, para la captación de las personas mayores de 85 años y en particular, de las centenarias, fue necesario contactar con trabajadoras sociales de base de diversos municipios de la provincia de Guipúzcoa.

La confidencialidad de los datos obtenidos ha sido asumida cumpliendo con la ley de protección de datos. Durante todo el proyecto se ha garantizado el cumplimiento de la Ley Orgánica 15/1999, de 13 de diciembre, de Protección de Datos de Carácter Personal (LOPD) y la Ley 41/2002, de 14 de noviembre, Básica Reguladora de la Autonomía del Paciente y de Derechos y Obligaciones en Materia de Información y Documentación Clínica. Este trabajo de investigación obtuvo la aprobación del Comité de Ética Asistencial de Ingema-Matia-Hurkoa.

\section{Análisis de datos}

Una vez efectuada la recogida de datos, se procedió al análisis estadístico, efectuado utilizando el paquete estadístico SPSS versión 21.0. Se utilizó un ANOVA y posteriormente se efectuaron pruebas post hoc Tukey de comparación múltiple.

\section{RESULTADOS}

En la variable afecto positivo, el ANOVA mostró un efecto significativo, $F(3,253)=20.84, p<0.001$ : el afecto positivo tendía a disminuir a medida que avanza la edad. Los análisis post hoc mostraron diferencias significativas del grupo de personas centenarias con los grupos de edad de 65 a 74 años y de 74 a 84 años, pero no con en el grupo de 85 a 94 años. Los valores descriptivos se presentan en la tabla 2.

En la variable afecto negativo, el ANOVA mostró un efecto significativo, $F(3,253)=4.63, p=0.004$ : el afecto negativo tendía a disminuir con la edad. En la prueba post hoc se observó que existían diferencias estadísticamente significativas del grupo de personas centenarias con el grupo de edad más joven (de 65 a 74 años), pero no con los dos grupos de edad intermedios (Tabla 3 ).

En la variable satisfacción con la vida, el ANOVA mostró un efecto significativo, $F(3,252)=2.90, p=0.036$ : la satisfacción con la vida tendía a aumentar con la edad. La prueba post hoc reveló solamente una diferencia significativa entre las personas centenarias y las de 65 a 74 años.

Con respecto a la variable soledad, el ANOVA mostró un efecto significativo, $F(3,241)=5.58, p=0.001$ : la soledad tendía a aumentar con la edad. La prueba post hoc solamente mostró diferencias estadísticamente significativas en el grupo de las personas 
centenarias con los dos grupos de edad más jóvenes. Las diferencias entre el grupo de 85 a 94 años y el de centenarios no alcanzó la significatividad estadística.

Tabla 2. Valores descriptivos de las variables emocionales: afecto positivo y negativo, satisfacción con la vida y soledad

\begin{tabular}{lcccc}
\hline \multicolumn{1}{c}{ Variable } & Rango de edad & $N$ & $M$ & $D T$ \\
\hline \multirow{3}{*}{ Afecto positivo } & $65-74$ & 75 & 31.23 & 7.02 \\
& $75-84$ & 80 & 26.80 & 5.68 \\
& $85-94$ & 69 & 24.10 & 6.43 \\
& $95-104$ & 33 & 22.82 & 5.79 \\
\hline \multirow{4}{*}{ Afecto negativo } & $65-74$ & 75 & 16.55 & 5.94 \\
& $75-84$ & 80 & 14.45 & 3.08 \\
& $85-94$ & 69 & 14.94 & 4.31 \\
Satisfacción con la vida & $95-104$ & 33 & 13.39 & 4.35 \\
& $65-74$ & 75 & 26.44 & 5.21 \\
& $75-84$ & 80 & 27.70 & 4.69 \\
& $85-94$ & 68 & 27.90 & 4.22 \\
Soledad & $95-104$ & 33 & 29.06 & 2.46 \\
\hline & $65-74$ & 71 & 27.00 & 5.79 \\
& $75-84$ & 79 & 26.86 & 6.07 \\
& $85-94$ & 67 & 28.70 & 6.25 \\
& $95-104$ & 28 & 31.79 & 5.94 \\
\hline
\end{tabular}

Tabla 3. Contraste de medias entre grupos de edad en afecto positivo y negativo, satisfacción con la vida y

\begin{tabular}{lccc}
\multicolumn{4}{c}{ soledad } \\
\hline \multirow{4}{*}{ Variable } & \multicolumn{2}{c}{ Rango de edad } & $p$ \\
\hline \multirow{3}{*}{ Afecto positivo } & $65-74$ & $95-104$ & 0,000 \\
\cline { 2 - 4 } & $75-84$ & $95-104$ & 0,048 \\
\cline { 2 - 4 } & $85-94$ & $95-104$ & 0,772 \\
\hline \multirow{3}{*}{ Afecto negativo } & $65-74$ & $95-104$ & 0,006 \\
\cline { 2 - 4 } & $75-84$ & $95-104$ & 0,676 \\
\cline { 2 - 4 } & $85-94$ & $95-104$ & 0,376 \\
\hline \multirow{3}{*}{$\begin{array}{l}\text { Satisfacción } \\
\text { con la vida }\end{array}$} & $65-74$ & $95-104$ & 0,030 \\
\cline { 2 - 4 } & $75-84$ & $95-104$ & 0,466 \\
\hline \multirow{3}{*}{ Soledad } & $85-94$ & $95-104$ & 0,619 \\
\cline { 2 - 4 } & $65-74$ & $95-104$ & 0,002 \\
\hline
\end{tabular}

A continuación, analizaremos los resultados relativos a las estrategias de regulación. En primer lugar, los resultados relativos a la regulación de la tristeza y en segundo lugar los que respectan a la ira. Los descriptivos se presentan en la tabla 4. 
ETXEBERRIA. Características emocionales de las personas mayores

Tabla 4. Valores descriptivos de las variables emocionales: estrategias de regulación

\begin{tabular}{|c|c|c|c|c|}
\hline Estrategia de regulación & Rango de edad & $N$ & $M$ & $S D$ \\
\hline \multicolumn{5}{|c|}{ Tristeza } \\
\hline \multirow{4}{*}{ Proactiva: Expresión } & $65-74$ & 75 & 15.57 & 4.93 \\
\hline & $75-84$ & 80 & 12.95 & 4.80 \\
\hline & $85-94$ & 69 & 11.06 & 4.57 \\
\hline & $95-104$ & 31 & 9.13 & 3.75 \\
\hline \multirow{4}{*}{ Proactiva: Búsqueda de consejo } & $65-74$ & 75 & 13.91 & 3.58 \\
\hline & $75-84$ & 80 & 12.85 & 3.98 \\
\hline & $85-94$ & 69 & 10.46 & 3.71 \\
\hline & $95-104$ & 31 & 8.10 & 3.27 \\
\hline \multirow{4}{*}{ Estrategias pasivas } & $65-74$ & 75 & 14.17 & 3.20 \\
\hline & $75-84$ & 79 & 15.25 & 3.86 \\
\hline & $85-94$ & 69 & 16.57 & 4.03 \\
\hline & $95-104$ & 31 & 17.77 & 4.01 \\
\hline \multirow{4}{*}{ Solución de problemas } & $65-74$ & 75 & 11.76 & 3.97 \\
\hline & $75-84$ & 80 & 10.75 & 3.87 \\
\hline & $85-94$ & 68 & 8.26 & 4.13 \\
\hline & $95-104$ & 31 & 6.03 & 2.97 \\
\hline \multicolumn{5}{|c|}{ Ira } \\
\hline \multirow{4}{*}{ Proactiva: Expresión } & $65-74$ & 75 & 16.48 & 4.55 \\
\hline & $75-84$ & 80 & 14.89 & 4.17 \\
\hline & $85-94$ & 69 & 11.62 & 4.60 \\
\hline & $95-104$ & 31 & 10.23 & 4.16 \\
\hline \multirow{4}{*}{ Proactiva: Búsqueda de consejo } & $65-74$ & 75 & 12.51 & 3.55 \\
\hline & $75-84$ & 80 & 11.64 & 3.42 \\
\hline & $85-94$ & 69 & 9.72 & 3.39 \\
\hline & $95-104$ & 31 & 8.16 & 3.52 \\
\hline \multirow{4}{*}{ Estrategias pasivas } & $65-74$ & 74 & 12.92 & 4.77 \\
\hline & $75-84$ & 80 & 13.68 & 5.04 \\
\hline & $85-94$ & 68 & 15.79 & 5.09 \\
\hline & $95-104$ & 31 & 15.03 & 6.17 \\
\hline \multirow{4}{*}{ Solución de problemas } & $65-74$ & 75 & 11.76 & 3.97 \\
\hline & $75-84$ & 80 & 10.75 & 3.87 \\
\hline & $85-94$ & 68 & 8.26 & 4.13 \\
\hline & $95-104$ & 31 & 6.03 & 2.97 \\
\hline
\end{tabular}

\section{Tristeza}

En la variable Estrategia proactiva: expresión, el ANOVA mostró un efecto significativo, $F(3,251)=18.44, p<0.000$ : la expresión emocional tendía a disminuir con la edad. La prueba post hoc mostró diferencias significativas del grupo de las personas centenarias con los grupos de 65 a 74 y de 75 a 84 años, pero no con el grupo de 85 a 94 años.

Con respecto a la variable Estrategia proactiva: búsqueda de consejo el ANOVA mostró un efecto significativo, $F(3,251)=23.26, p<0.000$ : la búsqueda de consejo tendía a disminuir con la edad. La prueba post hoc mostró diferencias del grupo de las personas centenarias con todos los grupos de edad más jóvenes.

En las Estrategias pasivas el ANOVA mostró también un efecto significativo, $F(3,250)=8.84, p<0.000$ : las estrategias pasivas tendían a aumentar con la edad. La prueba post hoc mostró diferencias significativas del grupo de las personas centenarias con los grupos de 65 a 74 y de 75 a 84 años, pero no con el de 85 a 94 años. 
ETXEBERRIA. Características emocionales de las personas mayores

Tabla 5. Contraste de medias entre grupos de edad en estrategias de regulación

\begin{tabular}{|c|c|c|c|}
\hline Estrategia de regulación & \multicolumn{2}{|c|}{ Rango de edad } & $p$ \\
\hline \multicolumn{4}{|c|}{ Tristeza } \\
\hline \multirow{3}{*}{ Proactiva: Expresión } & $65-74$ & $95-104$ & 0.000 \\
\hline & $75-84$ & $95-104$ & 0.001 \\
\hline & $85-94$ & $95-104$ & 0.226 \\
\hline \multirow{3}{*}{$\begin{array}{l}\text { Proactiva: Búsqueda de } \\
\text { consejo }\end{array}$} & $65-74$ & $95-104$ & 0.000 \\
\hline & $75-84$ & $95-104$ & 0.000 \\
\hline & $85-94$ & $95-104$ & 0.018 \\
\hline \multirow{3}{*}{ Estrategias pasivas } & $65-74$ & $95-104$ & 0.000 \\
\hline & $75-84$ & $95-104$ & 0.009 \\
\hline & $85-94$ & $95-104$ & 0.444 \\
\hline \multirow{3}{*}{ Solución de problemas } & $65-74$ & $95-104$ & 0.000 \\
\hline & $75-84$ & $95-104$ & 0.000 \\
\hline & $85-94$ & $95-104$ & 0.042 \\
\hline \multicolumn{4}{|l|}{ Ira } \\
\hline \multirow{3}{*}{ Proactiva: Expresión } & $65-74$ & $95-104$ & 0.000 \\
\hline & $75-84$ & $95-104$ & 0.000 \\
\hline & $85-94$ & $95-104$ & 0.460 \\
\hline \multirow{3}{*}{$\begin{array}{l}\text { Proactiva: Búsqueda de } \\
\text { consejo }\end{array}$} & $65-74$ & $95-104$ & 0.000 \\
\hline & $75-84$ & $95-104$ & 0.000 \\
\hline & $85-94$ & $95-104$ & 0.161 \\
\hline \multirow{3}{*}{ Estrategias pasivas } & $65-74$ & $95-104$ & 0.221 \\
\hline & $75-84$ & $95-104$ & 0.596 \\
\hline & $85-94$ & $95-104$ & 0.903 \\
\hline \multirow{3}{*}{ Solución de problemas } & $65-74$ & $95-104$ & 0.000 \\
\hline & $75-84$ & $95-104$ & 0.000 \\
\hline & $85-94$ & $95-104$ & 0.042 \\
\hline
\end{tabular}

Finalmente, y en lo que a la Solución de Problemas se refiere, el ANOVA señaló un efecto significativo, $F(3,250)=21.26, p<0.000$ : la solución de problemas tendía a disminuir con la edad. La prueba post hoc mostró diferencias significativas de las personas centenarias con todos los grupos de edad más joven (Tabla 5).

Ira

A continuación, se van a exponer los resultados obtenidos en la comparación de grupos en las estrategias de regulación de la ira.

En la variable Estrategia proactiva: expresión, el ANOVA mostró un efecto significativo, $F(3,251)=23.21, p<0.000$ : la expresión emocional tiende a disminuir con la edad. La prueba post hoc de Tukey indicó que existían diferencias significativas entre el grupo de centenarios con los grupos de 65 a 74 años y con el de 75 a 84 años, pero no con el grupo de 85 a 94 años.

En la variable Estrategia proactiva: Búsqueda de consejo, el ANOVA mostró igualmente un efecto significativo, $F(3,251)=15.63, p<0.000$ : la búsqueda de consejo tendía a disminuir con la edad. La prueba post hoc mostró que existían diferencias significativas, con los grupos de 65 a 74 años y de 75 a 84 años, pero no con el grupo de 85 a 94 años. 
En lo que respecta a las Estrategias pasivas, el ANOVA indicó que existía un efecto significativo, $F(3,249)=4.27, p=0.006$ : las estrategias pasivas tendían a aumentar con la edad. La prueba post hoc Tukey indicó que no existían diferencias significativas con ninguno de los tres grupos de edad más jóvenes.

Finalmente, y en lo que a la Solución de Problemas se refiere, el ANOVA mostró un efecto significativo, $F(3,250)=21.26, p<0.000$ : la solución de problemas tendía a disminuir con la edad. La prueba post hoc indicó que existían diferencias significativas en las personas centenarias con todos los grupos de edad más jóvenes.

\section{DISCUSIÓN Y CONCLUSIONES}

Los resultados revelaron en las personas centenarias, una disminución de la afectividad positiva y un aumento de la soledad en comparación con los grupos de 65 a 74 años y de 75 a 84 años, pero no con el de 85 a 94 años. Por otra parte, presentaban menor afecto negativo y más satisfacción con la vida, en comparación con el grupo de 65 a 74 años. Con respecto a las estrategias de regulación utilizan en menor medida las estrategias proactivas (expresión y búsqueda de consejo), al menos en comparación con los grupos de 65 a 74 años y de 75 a 84 años, en la regulación tanto de la tristeza como de la ira. También se observó que la utilización de estrategias pasivas (evitación, aceptación y supresión) aumentaba, en la regulación de la tristeza y en comparación con los grupos de edad mencionados. Sin embargo, este mayor uso de las estrategias pasivas no se daba en el caso de la ira. Con respecto a la solución de problemas, las personas centenarias utilizan en menor medida este tipo de estrategias en comparación con los grupos de edad más jóvenes.

En lo que respecta al afecto positivo, los resultados de este estudio concuerdan con los de investigaciones previas, que indican igualmente que el afecto positivo disminuye en las personas centenarias (Martin y cols., 2012). Aquí hay que decir que en nuestro estudio la diferencia entre los centenarios y el grupo anterior, aunque iba en la misma línea, no alcanzó la significatividad estadística.

En relación con el afecto negativo, los resultados del presente estudio concuerdan con los de investigaciones previas que indicaban que en los mayores de 85 años no existían diferencias significativas en afecto negativo (Staudinger y cols., 1999). Esta estabilidad de las emociones negativas se ha interpretado por algunos autores como resiliencia psicológica. La resiliencia en la vejez extrema se convertiría en la habilidad para utilizar los recursos disponibles para aceptar las inevitables pérdidas a nivel físico y psicosocial. En este sentido, sería una manera de evitar estados afectivos negativos, mientras que se buscarían situaciones y actividades significativas e importantes para la persona. 
Por tanto, tal y como concluyó Kunzmann (2000) el bienestar emocional en la vejez se perfila como una trayectoria no lineal, sino multidireccional. Esta autora argumenta que el declinar del afecto positivo en las personas mayores puede estar ligado a competencias objetivas, las cuales declinan con la edad, y que el afecto negativo no aumenta, sino que permanece estable porque se asocia con aspectos subjetivos que no cambian tanto con la edad.

En lo que respecta a la satisfacción con la vida, los resultados del presente trabajo indican una estabilidad de la satisfacción con la vida e incluso un cierto incremento en las personas centenarias (al menos, en comparación con el grupo más joven). El hecho de que se produzca cierta estabilidad o incluso cierto incremento en la satisfacción con la vida, pese a las circunstancias negativas (factores de salud y cercanía de la muerte), podría estar influenciado por la positividad en la memoria de los mayores. La evaluación cognitiva positiva del pasado que realizan los muy mayores puede que sea una estrategia adaptativa mediante la cual minimizan los sentimientos de insatisfacción (Aberg y cols., 2005). Esto podría deberse a que las personas que llegan a edades tan extremas, como se ha dicho, son más hábiles evitando las emociones negativas, mientras que buscan experiencias vitales emocionalmente significativas (Carstensen, Fung, y Charles, 2003).

En lo que a la soledad se refiere, estudios previos han hallado una asociación positiva entre la edad y la soledad (Long y Martin, 2000). Los resultados del presente estudio corroboran las conclusiones de dichos estudios ya que las personas centenarias eran las que más sentimientos de soledad manifestaban, siendo éstos significativamente diferentes a los de los grupos de 65 a 75 años y de 75 a 84 años.

A pesar de que la soledad puede tener muchas consecuencias negativas en el bienestar físico y psicológico, también puede tener una vertiente positiva, ya que dinamiza una reestructuración adaptativa y moviliza estrategias eficaces para afrontarla con éxito o incluso obtener beneficio de ella (Rubio y Aleixandre, 2001). Este tipo de planteamientos son congruentes con los resultados de la presente investigación, ya que, como se ha visto, el aumento del sentimiento de soledad en las personas centenarias no implicaría un aumento de su afectividad negativa.

Con respecto a las estrategias de regulación, los resultados del presente trabajo de investigación corroboran los obtenidos en la investigación previa (Poon y cols., 2010), ya que las personas centenarias afirmaron utilizar en menor medida las estrategias proactivas (expresión y búsqueda de consejo), al menos en comparación con los grupos de 65 a 74 años y de 75 a 84 años, en la regulación tanto de la tristeza como de la ira. También se observó que la utilización de estrategias pasivas (evitación, aceptación y supresión) aumentaba, en la regulación de la tristeza y en comparación con los grupos de edad mencionados. Sin embargo, este mayor uso de las estrategias pasivas no se daba en el caso de la ira. 
Estos resultados tienen pleno sentido, ya que, en comparación con las personas centenarias, los sexagenarios y septuagenarios pueden todavía participar en actividades sociales y actividades varias (Martin, Poon, Kim, y Johnson, 1996); en cambio, en las centenarias, las limitaciones funcionales, sensoriales y cognitivas podrían verse aumentadas y ello repercutir negativamente en la utilización de estrategias proactivas y la solución de problemas, estrategias que parecen requerir de una mayor capacidad cognitiva y funcional (Ochsner y Gross, 2005). Es decir, que se pueden utilizar diferentes tipos de estrategias de regulación en diferentes momentos del ciclo vital (Opitz, Rauch, Terry, y Urry, 2012).

Los sucesos críticos del envejecimiento puede que sean percibidos por las personas mayores como sucesos en los que no pueden intervenir o como situaciones que no permiten el cambio por su propia intervención. Ello explicaría que en las edades más avanzadas prevalezcan las estrategias pasivas sobre las activas. El afrontamiento o regulación pasivo podría considerarse adaptativo cuando la situación no permite cambios y solo cabe aceptarla (Baltes y Freund, 2003). Una menor implicación podría entenderse como un mecanismo de protección y a la vez como estrategia para mantener el bienestar emocional, lo cual puede entenderse nuevamente como una forma de favorecer la resiliencia (Etxeberria y cols., 2015).

La presente investigación abre un camino hacia el cuestionamiento del rol adaptativo de las estrategias proactivas en toda edad y circunstancia, sugiriendo que, en edades muy avanzadas y para la maximización del bienestar, las estrategias pasivas podrían ser más adaptativas.

En conclusión, y a pesar de que las limitaciones cognitivas, funcionales, de salud, etc. son más prevalentes entre las personas centenarias, esto no parece repercutir negativamente en su bienestar emocional, al contrario. En las personas centenarias, al menos en las que mantienen cierta autonomía, se observa que, a pesar de experimentar menos afecto positivo, y, asimismo, más soledad se produce cierta disminución en el afecto negativo, y cierto aumento de la satisfacción con la vida.

\section{REFERENCIAS}

Aberg, A.C., Sidenvall, B., Hepworth, M., O’Reilly, K., y Lithell, H. (2005). On loss of activity and independence, adaptation improves life satisfaction in old age -a qualitative study of patients' perceptions. Quality of Life Research, 14(4), 1111-1125.

Ailshire, J.A., y Crimmins, E.M. (2011). Psychosocial Factors Associated with Longevity in the United States: Age Differences between the Old and Oldest-Old in the Health and Retirement Study. Journal of Aging Research, 2011, 1-10.

Baltes, P.B., y Freund, A.M. (2003). Human strengths as the orchestration of wisdom and selective optimization with compensation. En L.G. Aspinwall y U.M. Staudinger, A psychology of human strengths: Perspectives on an emerging field (Eds), (pp. 23-35). Washington, DC: American Psychological Association. 
Baltes, P.B., y Mayer, K.U. (1999). The Berlin Aging Study: Aging from 70 to 100. New York: Cambridge University Press.

Etxeberria, I., Etxebarria, I., Urdaneta, E., y Yanguas, J.J. (2015). Age differences among older adults in the use of emotion regulation strategies. What happens among over $85 \mathrm{~s}$ and centenarians? Aging and Mental Health, doi: 10.1080/13607863.2015.1050995

Galiana, L., Gutiérrez, M., Sancho, P., Oliver, A., y Tomás, J.M. (2015). Propiedades psicométricas de la versión española de la Escala Temporal de Satisfacción con la Vida (Temporal Satisfaction With Life Scale, TSLS): Un estudio en mayores que acuden a programas universitarios. European Journal of Investigation in Health, Psychology and Education, 5(3), 335-344.

Holzschuher, H. (1984). Mir fällt die decke auf den kopf"alter [I feel really shut in: About the experi-ence of loneliness in old age]. (Kölner Verlag). Berlin, Germany.

Kunzmann, U. (2008). Differential age trajectories of positive and negative affect: Further evidence from the Berlin Aging Study. Journals of Gerontology Series B: Psychological Sciences and Social Sciences, 63, 261-270.

Long, M.V., y Martin, P. (2000). Personality, Relationship, Closeness, and Loneliness of Oldest Old adults and Their Children. Journals of Gerontology: Psychological Sciences, 55(5), 311-319.

Martin, P., Da Rosa, G., Margrett, J., Garasky, S., y Franke, W. (2012). Stability and change in affect among centenarians. International Journal of Aging and Human Development, 75(4), 337-349.

Martin, P., Kliegel, M., Rott, C., Poon, L., y Johnson, M. (2008). Age differences and changes of coping behavior in three age groups: Findings from the Georgia Centenarian Study. Journal of Aging and Human Development, 66, 97-114.

Montero, P., López-Giménez, M.R., Acevedo, P, y Mora, A.I. (2015). Envejecimiento saludable: perspectiva de género y de ciclo vital. European Journal of Investigation in Health, Psychology and Education, 5(1), 55-63.

Mroczek, D.K., y Spiro, A. (2005). Change in life satisfaction during adulthood: findings from the veterans affairs normative aging study. Journal of Personality and Social Psychology, 88(1), 189-202.

Ochsner, K.N., y Gross, J.J. (2005). The cognitive control of emotion. Trends in Cognitive Sciences, 9(5), 242-249.

Opitz, P.C., Rauch, L.C., Terry, D.P., y Urry, H.L. (2012). Prefrontal mediation of age differences in cognitive reappraisal. Neurobiology of Aging, 33, 645-655. doi:10.1016/j.neurobiolaging.2010.06.004

Pinquart, M., y Sörensen, S. (2001). Influences on loneliness in older Adults: A Meta Analysis. Basic and Applied. Social Psychology, 23, 245-66.

Poon, L.W., Martin, P., y Margrett, J. (2010). Cognition and emotion in centenarians. En C.A. Depp y D.V. Jeste (Eds.), Successful cognitive and emotional aging. Arlington, VA: American Psychiatric Publishing.

Rubio, R., y Aleixandre, M. (2001). Un estudio sobre la soledad en las personas mayores: entre el estar solo y el sentirse solo. Revista Multidisciplinar de Gerontología, 11(1), $23-28$.

Sandín, B., Chorot, P., Lostao, L., Joiner, T., Santed, M., y Valiente, R. (1999). Escala PANAS de afecto positivo y negativo: validación factorial y convergencia transcultural. Psicothema, $11(1), 37-51$.

Staudinger, U.M., Freund, A., Linden, M., y Maas, I. (1999). Self, personality, and life regulation: Facets of psychological resilience in old age. En P.B. Baltes y K.U. Mayer (Eds.), The 
Berlin Aging Study: Aging from 70 to 100 (pp. 302-328). New York: Cambridge University Press.

Vázquez, A.J., y Jimenez, R. (1994). Escala de soledad UCLA revisada. Fiabilidad y validez de una versión española. Revista de Psicología de La Salud, 6(1), 45-54.

Yanguas, J.J., Prieto, D., Urdaneta, E., Garcia, A., Buiza, C., y Etxeberria, I. (2008). El positivismo de la vejez: análisis cualitativo de las emociones en las personas mayors. Revista Española de Geriatría y Gerontología, 43(1), 154.

Yi, Z., y Vaupel, J.W. (2002). Functional capacity and self evaluation of health and life of oldest old in China. Journal of Social Issues, 58(4), 733-748.

Recibido: 5 de mayo de 2015

Recepción Modificaciones: 18 de enero de 2016

Aceptado: 20 de enero de 2016 